\title{
DeR SCHNITZELBANK-SONG UND SEINE REZEPTION IN DEN USA
}

\author{
Fabian Bade
}

\section{Prolog}

Deutschsprachiger populärer Musik fällt in den USA trotz eines Bevölkerungsanteils von etwa $15 \%$ US-Amerikanern mit deutschen Wurzeln ${ }^{1}$ allenfalls eine marginale Rolle zu. Dies zeigt sich sowohl im sporadischen Auftreten weniger Stücke in den Billboard-Charts ${ }^{2}$ als auch bei der geringen Anzahl von Songs, die in anderen Kontexten wie Werbespots ${ }^{3}$ oder Kinofilmen ${ }^{4}$ Eingang in die US-amerikanische Popkultur fanden. Nimmt man die Zeit nach dem Ende des Zweiten Weltkrieges in den Blick, so gehören Kraftwerk, Trio, Nena, Falco und Rammstein samt einiger heute dort weniger bekannter Interpreten wie bspw. Marlene Dietrich und Peter Schilling zu den zentralen Repertoirequellen deutschsprachiger (Pop-)Musik in den Vereinigten Staaten.

Bei genauerer Betrachtung fällt hier allerdings ein weiterer Titel ins Auge, der in verschiedenen Zusammenhängen immer wiederkehrt und um den es im Folgenden gehen soll: die Schnitzelbank. Interessanterweise ist dieser Song

1 Vgl. den Bericht des U.S. Census aus dem Jahr 2000, https://www.census. gov/history/pdf/ancestry.pdf, S. 3, Zugriff: 5.4.2018.

2 Vgl. etwa die Songs »Da Da Da« (Trio, 1982, Platz 33 der Billboard Dance Charts), »99 Luftballons « (Nena, 1984 auf Platz 2 der Billboard Hot 100) oder »Rock Me Amadeus« (Falco, 1986, Platz 1 der Billboard Hot 100).

3 Trios »Da $\mathrm{Da} \mathrm{Da}$ « wurde bspw. in der Originalversion von 1982 in TV-Werbespots zur Hauptsendezeit im Rahmen großer medialer Ereignisse eingesetzt. 1997 findet sich der Titel in einem Volkswagen-Clip (vgl. Robert Peele am 4. Dezember 2009 in der New York Times unter https://wheels.blogs. nytimes.com/2009/12 /04/classic-ad-volkswagen-golf/, Zugriff: 4.4.2018) und noch einmal neun Jahre später in einer von Christina Aguilera gesungenen Version innerhalb eines Werbespots zur Fußball-Weltmeisterschaft 2006 für Pepsi (vgl. Schock, Axel am 4.12.2012 in einem Beitrag in der Mitteldeutschen Zeitung: https://www.mzweb.de/kultur/musik--trio----von-grossenkneten-in-die-weite-welt-729750, Zugriff: 4.4.2018.

4 So wurden etwa die Rammstein-Songs »Heirate mich« (1995) und »Rammstein « (1995) im Film Lost Highway des Regisseurs David Lynch verwendet. 
- präziser formuliert: sind die Schnitzelbank-Songs, denn der Song tritt in verschiedenen Phänotypen auf - nicht nur wesentlich älter als die angeführten Beispiele, sondern in Deutschland - wenn überhaupt - nur wenigen Menschen bekannt. Eher könnte man sagen, dass hierzulande nicht die Schnitzelbank als Song, sondern ein Schnitzelbank-Sujet bekannt ist - dies aber in der Regel in anderen Nexus.

\section{Was genau meint der Terminus Schnitzelbank?}

Eine Schnitzelbank ist laut Duden zunächst einmal ein veraltetes Wort für eine Werk- oder Schnitzbank. ${ }^{5}$ Zudem bezeichnet der Begriff je nach Region in Europa und den USA auf den ersten Blick scheinbar völlig zusammenhangslose kulturelle Traditionen, die im Kontext mit (Hochzeits-)Festen, alemannischen Fastnachtstraditionen und Trinkliedern stehen. Hier meint der Terminus Schnitzelbank primär einen Sujetaufgriff innerhalb US-amerikanischer Popsongs. Das Sujet des Kettenliedes Schnitzelbank hat sich aus einem Volkslied heraus entwickelt, welches wiederum textlich Bezug auf die genannten Brauchtümer nimmt und über deutsche Auswanderer in die USA gelangte. Wie bei Volksliedern nicht unüblich, liegen alleine in den Vereinigten Staaten zahlreiche Varianten der Schnitzelbank vor. ${ }^{6}$ Die Zusammenhänge zwischen den europäischen Vorläufern und dem in den USA heute bekannten Volkslied sind bereits dokumentiert und in Teilen beschrieben worden (vgl. Keel 2002: 21ff., Keel 2003: 221ff. sowie Keel 2009: 213ff.). Von besonderem Interesse ist die Tatsache, dass das Schnitzelbank-Sujet auf verschiedene Arten Eingang in die US-Popkultur fand (vgl. Punkt 6 dieses Aufsatzes), u.a. durch die Verwendung in Popsongs. Bevor dies konkret in den Blick genommen werden soll, lohnt aber eine Betrachtung der vorausgegangenen Rezeption deutschsprachiger Musik in den USA sowie eine kurze Beschreibung der zugrunde liegenden Mechanismen.

5 Vgl. https://www.duden.de/rechtschreibung/schnitzelbank, Zugriff: 15.4.2018.

6 Vgl. exemplarisch eine Version von John Schmid im Pennsylvania Dutch-Dialekt (2004) unter https://www.youtube.com/watch?v=lvlefmFEu2U\&frags=pl\%2Cwn, Zugriff: 9.8.2018. 


\section{Die Rezeption deutscher und deutschsprachiger Musik in Nordamerika}

Hadamer (2001: 119f.) beschreibt gängige musikkulturelle Praxen des späten 18. und 19. Jahrhunderts und erklärt, wie religiöse und weltliche deutschsprachige Lieder Eingang in die Musikkultur Nordamerikas fanden. Er macht im Hinblick auf den Sprachwechsel bei Liedern sechs Verfahrensweisen aus (1. wörtliche Übersetzungen, 2. freie Nachahmungen, 3. Parodien, 4. neue Textkreationen zu bestehenden Melodien, 5. das Ersetzen deutscher durch englische Texte und 6. das Zusammenfügen deutscher Melodien mit deutschen Texten in englischen Übersetzungen) und spricht auch dann noch von einer »Form der Zweisprachigkeit«, wenn die Melodienamen trotz der Verwendung der englischen Sprache noch in deutscher Sprache angegeben werden (ebd.: 122). Seine Schlussfolgerung lautet, dass auf diese Weise ein überwiegend englischsprachiges Publikum Zugang zu deutschen und deutschsprachigen Liedern finden konnte, was wiederum zur Folge hatte, dass ein umfangreiches Repertoire deutscher Melodien als Vergleichsmaterial vorhanden ist, an dem US-Amerikaner »ihre Vorstellungen von deutschen Einwanderern und Deutsch-Amerikanern verhandeln konnten« (Hadamer 2008: 315). Dies ist insofern von Bedeutung, als dass sich hier andeutet, auf welch vielfältige Weisen musikkultureller Austausch im Zusammenhang mit deutschsprachiger Musik schon im 19. Jahrhundert stattgefunden haben muss. Hadamer beschreibt die Blütezeit der »freie[n] Adaptionspraxis« in den 1830er Jahren, innerhalb der Melodien unterschiedlichsten Ursprungs vom Tanzstück bis zur Opernarie für Sonntagsschulen und andere Anwendungsbereiche umfunktioniert worden sind und wie diese »neuen Lieder « in der populären Musik der USA aufgehen (Hadamer 2001: 127).

Im Folgenden sollen zunächst die europäischen Schnitzelbank-Traditionen kurz beschrieben werden, um Zusammenhänge mit dem Sujet in US-amerikanischen Popsongs zu verdeutlichen. In einem weiteren Abschnitt soll dann auf den Schnitzelbank-Song eingegangen werden, wie er heute in den USA als Volkslied kursiert - denn in dieser Form hat er mutmaßlich als Vorlage für viele Songs gedient. Anschließend wird das Vorkommen des SchnitzelbankSujets in US-Popsongs dargelegt. Die Grundlage hierfür bieten die BillboardCharts, deren Daten ausgewertet und tabellarisch dargestellt werden. Zum Abschluss sollen weitere Beispiele aus einem nicht im Billboard-Magazin erfassten Song sowie aus Zeitungen, Printwerbungen und Veranstaltungsankündigungen dabei helfen, die Schnitzelbank als deutsch-amerikanisches Phänomen innerhalb der Popkultur der Vereinigten Staaten zu verstehen. 


\section{Zu den europäischen Vorläufern der US-amerikanischen Schnitzelbank}

In der europäischen Geschichte sind mehrere Vorläufer bekannt, die auffällige Gemeinsamkeiten mit der heute in den USA geläufigen Schnitzelbank aufweisen. Parallelen lassen sich konkret in drei Fällen beobachten:

1. Im Moritaten- und Bänkelsängertum des Mittelalters im deutschsprachigen europäischen Raum,

2. bei der Verwendung des Schnitzelbank-Sujets innerhalb alemannischer Fastnachtsbrauchtümer in der Schweiz und Südwestdeutschlands und

3. in einem überlieferten Hochzeitsbrauchtum deutscher Siedler im heutigen Polen.

Im ersten und letzten Fall erlauben historische Quellen einen Blick auf die heute in der Form nicht mehr fortgeführten Traditionen; die Verwendung der Schnitzelbank innerhalb verschiedener Karnevalstraditionen lässt sich aber z.B. in Basel und Ellwangen noch heute beobachten. Folgende Prinzipen der drei genannten (musik-)kulturellen Traditionen können als wesentliche Eigenarten festgehalten werden, auch wenn nicht bei jeder Ausprägung alle Attribute gleichermaßen beobachtbar sind:

1. Bildtafeln werden in allen Traditionen - im Bänkelsang, bei dem genannten Hochzeitsbrauchtum deutscher Siedler und in bestimmten Ausprägungen der alemannischen Fastnacht - als zentrales Merkmal genutzt. Die Textinhalte werden gemeinhin in Form von einfachen Darstellungen und Skizzen veranschaulicht. Allen drei Traditionen ist gemein, dass die bildliche Darstellung eine Deutung durch Analphabeten möglich macht. Dies gilt zwar weniger für die kritischen und teilweise komplexeren Inhalte der Verse einiger Fastnachtstraditionen, jedoch ist die bildliche Illustration zentraler Momente in jedem Fall hilfreich und ermöglicht schreibunkundigen Personen kulturelle Teilhabe.

2. Die Aufführung findet in der Regel im Rahmen geselliger Anlässe statt. Dies trifft auf alle Traditionen zu.

3. Es gibt eine ausgeprägte Hierarchie und eine klare Aufgabenverteilung zwischen Vor- und Nachsängern: Im Hochzeitsbrauchtum wird nach dem Call and Response-Prinzip gesungen, in der alemannischen Fastnachtstradition dagegen singen die Nachsänger lediglich eine Refrainzeile: »Ei du schöne Schnitzelbank«. Im Umfeld des Bänkelsangs verhält es sich noch einseitiger: Der Bänkelsänger ist der aktive Part, die Rezipienten haben rein passiv am Geschehen teil. 
4. In einigen Fällen (Hochzeitsbrauchtum) liegt eine Kettenliedstruktur vor.

5. Eine Refrainzeile wie etwa »Ei du schöne Schnitzelbank« oder »Oh du schöne Schnitzelbank« ist bei der Schnitzelbank als Hochzeitsbrauchtum und Fastnachtsbrauch üblich - wenn auch häufig in leicht abgewandelter Form. Aufgrund der Passivität der Rezipienten gibt es im Bänkelsang im Regelfall keine Refrainzeile.

6. Trotz der typischerweise geselligen Kontexte, in denen alle Traditionen stehen, gehören auch Belehrung und Moral zu den Kennzeichen von Schnitzelbänken. Im Falle des Bänkelsangs und der Fastnachtstraditionen ist dies besonders ausgeprägt und zwar auch dann, wenn die Belehrung in unmittelbarem Zusammenhang mit Komik steht.

Um später beurteilen zu können, welche Attribute nun konkret auf einen Zusammenhang mit der US-amerikanischen Schnitzelbank deuten, werden die genannten Traditionen kurz beschrieben:

\subsection{Moritaten- und Bänkelsängertum und die Parallelen zur Schnitzelbanktradition in den USA}

Bänkelsänger transportieren im zentraleuropäischen Raum vom Mittelalter bis weit ins 19. Jahrhundert in der Regel dramatische Inhalte in Form von Liedern, wobei hier eher von einem Erzählen zu optionaler musikalischer Begleitung mit wechselndem Instrumentarium ausgegangen werden kann. Der Bänkelsänger steht dabei im Zentrum des Geschehens und deutet mit einem Stab auf Zeichnungen auf einer Tafel oder ähnlichen Gegenständen, die er zur Illustration der gesprochenen und gesungenen Inhalte nutzt (vgl. Abb. 1). Petzoldt sieht als konstituierende Elemente des Bänkelsangs vier zentrale Funktionskategorien - Belehrung, Sensation, Information und Unterhaltung - an, wobei mit der Zeit zunehmend eine Reduktion auf das Moment der Unterhaltung beobachtbar sei (vgl. Petzoldt 1978: 1ff.). Möglicherweise erkannte er bereits 1978 wichtige Anhaltspunkte für die unterschiedlichen Schnitzelbank-Ausprägungen und differierende Rezeptionsformen in Deutschland und den USA: Er argumentiert mit Blochs Begriff der Ungleichzeitigkeit von 1935 und verweist auf den Umstand, dass es zum gleichen Zeitpunkt durchaus zu unterschiedlichen musikkulturellen Entwicklungen und Wahrnehmungen verschiedener gesellschaftlicher Schichten kommen kann. Demzufolge träfen »Moralauffassung, Stoffe, Stil [und] Aussage« (ebd.: 2) aus vergangenen Zeiten auf ein ungleichzeitiges Publikum; eine potentielle Rezipienten-Gruppe also, die sich aus verschiedenen gesellschaftlichen Schichten 
rekrutiert und in ihrem Denken und Handeln keinesfalls als gleichmäßig progressiv eingestuft, sondern vielmehr als heterogen charakterisiert werden kann. Aus Ermangelung an Rezipienten, die noch in der Lage wären, ursprüngliche Kontexte zu verstehen, zieht er deshalb die logische Konsequenz: die »Überbetonung des unterhaltenden Charakters« (ebd.: 1) bleibt zurück, wenn ein Inhalt aufgrund zeitlicher Diskrepanz zwischen Konzept und Perzept analog zu den eingangs erwähnten Funktionskategorien schlichtweg nicht mehr als belehrend, sensationell oder informativ empfunden werden kann. Das Moment der Unterhaltung, die komische Seite der Schnitzelbank ist das, was in den USA heute vorrangig übrig geblieben ist von den facettenreichen Varianten europäischer Schnitzelbänke. Doch dazu später mehr.

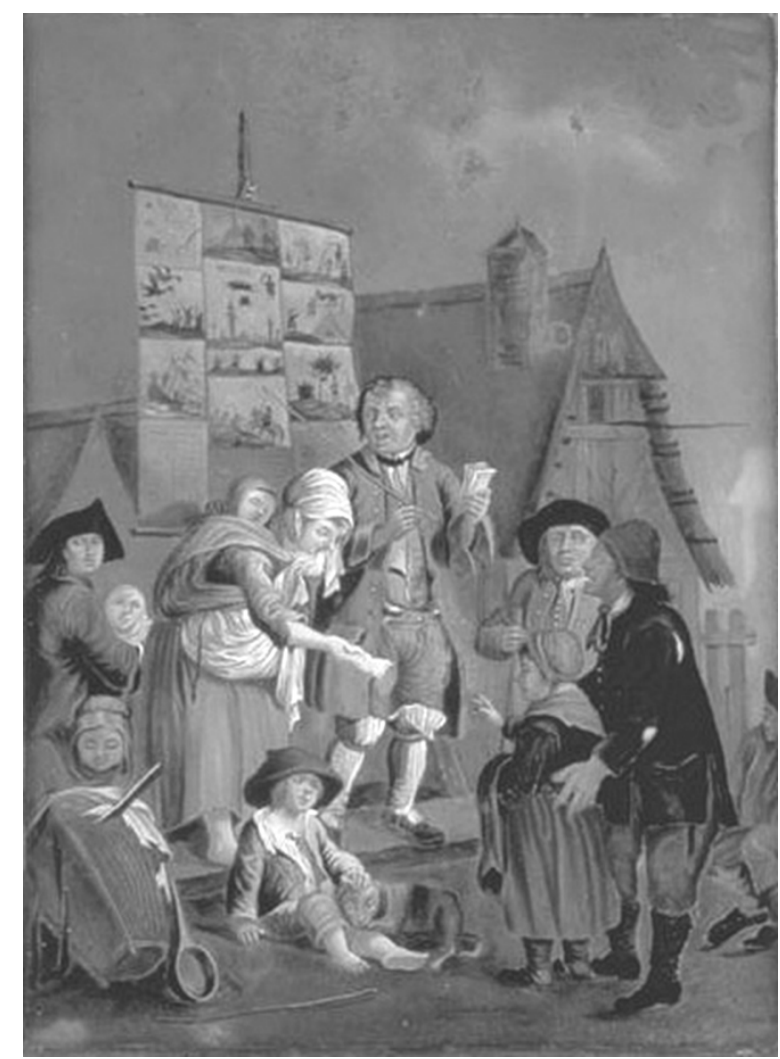

Abb. 1: Bänkelsänger mit Frau und ländlichem Publikum. Beromünster, etwa 17901800. Eglomisé auf Glas, Schweizerisches Landesmuseum, Zürich. Original in Farbe.

\subsection{Die Schnitzelbank als Hochzeitsbrauchtum}

Aus verschiedenen Gebieten in Zentraleuropa, von der Halbinsel Hel in Polen bis nach Winningen an der Mosel, liegen Überlieferungen von Hochzeitsbräuchen vor, die textlich eine große Nähe zum Schnitzelbank-Song aufweisen, wie sich anhand des nächsten Beispiels zeigen lässt. Bereits in der Mitte des 
19. Jahrhunderts dokumentieren Volkskundler ein angeblich Jahrhunderte altes Brauchtum, das damals unter dem Terminus Lichtputzschere geläufig war (vgl. Mannhardt: 1855). ${ }^{7}$ Fischer aus dem Raum Danzig führten zu Hochzeiten ein halb improvisiertes Singspiel auf, bei dem eine Lichtputzschere einen Phallus symbolisiert. Ein männlicher Vorsänger illustriert während des Singens die Inhalte auf einem Tisch oder ähnlichen Gegenständen. Die von ihm gestellten Fragen wie »Ist das nicht ein kurz und lang « werden von den anwesenden Jungfrauen, die sich um den Vorsänger versammeln, postwendend mit »Ja, das ist ein kurz und lang « beantwortet. Im Gegensatz zur BänkelsängerTradition verfügt der Vorsänger hier nicht über eine fertige Bildtafel, sondern muss diese in Echtzeit zum Gesang erstellen. In jeder Strophe gibt es ein Reimpaar und bei jeder weiteren Strophe werden alle vergangenen Paare wiederholt sowie ein neues hinzugefügt. Die Reimpaare aus Mannhardts Beispiel lauten »storchenschnabel, mistelgabel, hühnersteig, harf' und geig', baum und mist, hafkeschiß, vater und sohn, Absalon, jud im haus, kukuk kukt zum Fenster raus, reiter zu pferd, feuerheerd, finger und ring, kleines kind, beil und klotz, bauernf-tz (vulva) (Mannhardt 1855: 259-261). Nach dem Aufzeichnen aller Begriffe beschließt der Vorsänger das Lied mit der Zeichnung eines Phallus-Symbols, das er mit seinen Händen bedeckt und hierzu singt: »Ein Hin und Her, eine Lichtputzscher! « Alle Frauen versuchen daraufhin, die Hände des Zeichners von dem Symbol loszureißen, ohne dabei die Skizzen zu beschädigen und singen währenddessen »Eine schöne, schöne Lichtputzscher « (ebd.). Dem Aberglauben zufolge wird die Ehe umso kinderreicher, wenn dies zügig gelingt. ${ }^{8}$ Obgleich diese Wortpaare nur geringfügige Übereinstimmungen mit der heute in den USA bekannten Version des Schnitzelbank-Songs aufweisen, wird die strukturelle Anlage als Kettenlied dennoch unmittelbar

7 Lichtputzschere ist eine heute kaum noch gebräuchliche Bezeichnung für eine Dochtschere. Vor allem in Europa war die Schnitzelbank in gesungener Form in vergangenen Jahrhunderten auch unter dem Titel Lichtputzschere, Hobelbank oder Snydersbank bekannt. Häufig zwar mit regional unterschiedlichen Schreibweisen, jedoch immer mit gleichen Ordnungsprinzipien als Kettenlied sowie mit mehreren begrifflichen Übereinstimmungen, wie kurz und lang - Hobelbank hin und her - Lichtputzscher.

8 Für eine präzise Darstellung dieses Brauchtums siehe Mannhardt (1855: 259-261). Interessant ist in dem Zusammenhang auch die Aussage, dass es sich bei den erwähnten Fischern um »seit jahrhunderten in abgeschiedenheit lebende [...] fischer [...] deutscher Abkunft« handelt, was darauf hindeutet, dass diese Hochzeitstradition bereits wesentlich länger bestand, als der Zeitpunkt ihrer schriftlichen Dokumentation durch die Wissenschaft in der Mitte des 19. Jahrhunderts vermuten lässt. 
deutlich. Als weitere Gemeinsamkeiten können zudem das hohe Maß an Vulgarität, die Bildtafel (auch wenn sie hier erst entsteht) sowie das Call and Response-Prinzip festgehalten werden.

Auch wenn der Begriff Lichtputzschere kaum noch in den heute in den USA geläufigen Versionen der Schnitzelbank vorkommt, sei erwähnt, dass es sehr wohl dokumentierte Einzelfälle gibt, die zeigen, dass der Begriff auch in zentraler und titelgebender Funktion zu finden ist: Korson (1949: 80f.) zeichnet 1937 in Rehrersburg, Pennsylvania ein Lied mit dem folgenden Text auf: »Ei du scheene, ei du scheene, ei du scheene Lichtputzscher. Is des net des Hie und Her? Ja des is des Hie und Her! Des Hie und Her, un die Lichtputzscher.« Dieses Beispiel zeigt deutlich, dass die Schnitzelbanktradition auch unter dem Terminus Lichtputzschere in den USA bekannt gewesen sein muss.

\subsection{Zur Verwendung des Schnitzelbank-Sujets innerhalb alemannischer Fastnachtsbrauchtümer in der Schweiz und Südwestdeutschlands}

Die dritte europäische Tradition, die Gemeinsamkeiten mit dem US-amerikanischen Schnitzelbank-Song aufweist, ist den alemannischen Fastnachtsbrauchtümern zuzuordnen. Ellwangen und Basel sind diejenigen Städte, in denen diese bis heute am deutlichsten ausgeprägt sind und sichtbar werden. In Ellwangen führen Tübinger Jurisprudenz-Referendare Mitte des 19. Jahrhunderts eine Schnitzelbank-Tradition ein. Die angehenden Juristen sichern sich die Unterstützung junger Gymnasiasten, die diesen Brauch bis heute fortführen. Der Übernahme und Konservierung dieser Tradition durch die Gymnasiasten verdankt sich auch der Name: Der Pennäler Schnitzelbank. Konkrete Inhalte sind kurze Spottverse, die sich mehrheitlich mit Verfehlungen der Lokalprominenz befassen ${ }^{9}$, teilweise aber auch nationale Entwicklungen aufgreifen und politische Kommentare beinhalten. ${ }^{10}$ Obwohl sich bereits in antiken Vorstellungen und Abläufen des Saturnalienfestes Prinzipien erkennen lassen, die hier konkret Anwendung finden ${ }^{11}$, ist eher anzunehmen, dass der

9 Ein Beispiel hierfür ist eine Schnitzelbank aus dem Jahr 1981, die die Abwesenheit des Bischofs bei der Firmung sowie die Haltung des ehemaligen Stadtpfarrers und Kreisdekans Patriz Hauser thematisiert: »Da kann ich's gleich selber machen, hört man Hauser spöttisch lachen, firmte doch in diesem Jahr nur ein Domkapitular « (Kohring/Nußbaumer/Hurler 2000: 173).

101936 ist ein kritischer Kommentar zur NS-Hetzschrift »Flammenzeichen « Inhalt eines Verses: »Nörgler, jetzt müsst ihr erbleichen! Denn es leuchten Flammenzeichen, Überall hin dringt ihr Schein, Holen selbst den letzten ein!« (Kohring/ Nußbaumer/Hurler 2000: 165).

11 Im Rom der Antike tauschten Sklaven und Herren während des Saturnalienfestes für einen Tag die Rollen. 
demokratische Geist der 1848er Generation in der Fastnacht einen Moment findet, im Schutze der Narrenfreiheit der Obrigkeit einen Spiegel vorzuhalten (vgl. Kohring 2000: 14). Da der Fokus der Kritik im Regelfall auf lokalen Geschehnissen liegt, ist in einer kleinen Stadt wie Ellwangen die Anonymität der Protagonisten essentiell für die Erhaltung des Brauchtums - und die Vermummung eine logische Konsequenz. Die in Schwarz gehüllte Exekutive der Schnitzelbank nennt sich in Ellwangen dementsprechend die Schwarze Schar.

Auch wenn dies auf andere Art und Weise geschieht: In Basel nimmt die Schnitzelbank gleichsam eine wesentliche Rolle im lokalen Fastnachtsgeschehen ein. Auch in der Basler Ausprägung spielt die Anonymität eine große Rolle. Der Schnitzelbank - dort in maskuliner Form - wird von Vorsängern präsentiert, die zumeist rhythmisch gesprochene oder gesungene Verse vortragen. Im Gegensatz zum Brauchtum aus Ellwangen sind die Ausführenden hier nicht komplett vermummt, tragen aber unterschiedliche und oftmals bunte Verkleidungen samt einer Larve (= Maske) und präsentieren mit einem Zeigestock die Inhalte der Verse auf einer Bildtafel, deren einzelne Zeichnungen hier Helgen genannt werden. ${ }^{12}$ So wie in Ellwangen das Spiegelbild von Handlungen zum Ausgangspunkt der Versdichtungen wird, kann dies auch hier als zentrales Moment bezeichnet werden. Fürstenberger und González (2002: 8) schrieben der Basler Schnitzelbangg ${ }^{13}$ die Funktion eines »Stadtspiegels [zu, in dem sich] das politische, soziale, aktuelle Umfeld, das sogenannte Spiegelbild [zeige]«. Die Funktion einer Widerspiegelung von Handlungen und Konsequenzen hat in Basel wiederum eine lange Tradition: Auf literarischer Ebene kritisierten schon Konrad von Würzburg in Die Klage der Kunst in der Mitte des 14. Jahrhunderts (vgl. Joseph 1885: 1ff.), Sebastian Brandt 1494 im Narrenschyff sowie Erasmus von Rotterdam in Lob der Torheit aus den Jahren 1511-1515 mangelnde Selbstreflexion oder auch Teilnahmslosigkeit am Kunstgeschehen (vgl. Fürstenberger und González 2002: 10ff.). Möglicherweise ist also auch die Basler Tradition älter, als die Dokumentation durch die Wissenschaft es bisher belegen kann. Durch das Spiegelbild wird auch eine Nähe zur ersten Bänkelsang-Funktionskategorie Petzoldts, der Belehrung, deutlich und könnte auf eine gemeinsame historische Vergangenheit hindeuten.

Auch wenn heutige Aufführungspraxen von Schnitzelbängg deutlich von denen aus den USA abzugrenzen sind, so weist doch einiges auf gemeinsame Vorläufer hin. Die wahrscheinlich bedeutendste Gemeinsamkeit ist der Umstand, dass in früheren Basler Ausprägungen eine Kettenliedstruktur vorlag,

12 Der Begriff Helgen leitet sich von den Heiligenbildern der Franziskaner ab; vgl. Fürstenberger/González (2002: 20).

13 Häufige alternative Schreibweise im Baseldeutschen Dialekt: Schnitzelbangg (Singular) resp. Schnitzebängg (Plural). 
auch wenn das in heutigen Versdichtungen der Schnitzelbank kaum noch der Fall ist. ${ }^{14}$ Die Überschneidungen sind allzu auffällig, als dass die Verbindungen zwischen den Traditionen ignoriert werden könnten: »Isch das nit e Spalethor? Jo das isch en-Eselsohr, Eselsohr, Spalethor. Ei du scheene, ei du scheene, Ei du scheene Schnitzelbangg. Isch das nit e Lindemaier? Jo das sin drei Oschteraier, Oschteraier, Lindemaier, Spalethor, Eselsohr. Ei du scheene, ei du scheene Ei du scheene Schnitzelbangg «. ${ }^{15}$

So eindeutig die Kettenliedstruktur hier erkennbar ist, so unterschiedlich ist die sprachliche Ausdrucksweise. Das Textverständnis wird nicht nur durch einen Dialekt, sondern auch durch Begriffe erschwert, die nur Personen zugänglich sind, die mit bestimmten Facetten des Basler Stadtlebens vertraut sind $^{16}$ (vgl. exemplarisch auch Canova 2005: 17). Dieses Lokalkolorit ist der deutlichste Unterschied zur US-Variante und der Hochzeitstradition, in der Inhalte prinzipiell unabhängig von der Region erhalten geblieben sind. Ein möglicher Grund für das spätere Aussterben der Kettenliedstruktur kann darin gelegen haben, dass sich die Schnitzelbank-Texte im Verlauf des 19. Jahrhunderts zunächst verlängerten, da Jahr für Jahr neue Strophen hinzugefügt wurden. Da allerdings erwartet wurde, dass die Verse eine gewisse Aktualität aufweisen, ist nachvollziehbar, dass Veraltetes durch Aktuelleres ersetzt wurde und sich die Kettenliedstruktur irgendwann aufweichte, verschwand und ersetzt wurde.

\section{Der Schnitzelbank-Song als Volkslied in den USA}

In den USA hat sich eine Schnitzelbanktradition durchgesetzt, die folgende Merkmale aufweist: Es handelt sich um ein gesungenes Volkslied, zu dessen Gesang üblicherweise eine Bildtafel Inhalte transportiert. Es besteht weiterhin eine klare Hierarchie zwischen Vorsänger und Nachsänger, das Call and

14 Der Basler Schnitzelbank-Experte Bernhard Batschelet bestätigte in einem Interview am 8. September 2016, dass die Refrainzeile in vielen Schnitzelbänken der Nachkriegszeit regelmäßig gesungen wurde. Heute ist diese Praxis im Basler Raum laut Batschelet aber fast ausgestorben. Vgl. exemplarisch die Darstellung Gernys in der Neuen Zürcher Zeitung, die regelmäßig zur Basler Fasnacht über die Schnitzelbank-Aufführungen berichtet, Gerny (2018) sowie die Darstellungen der Refrainzeile bei Röhrich/Brednich (1965: 524ff.) für den deutschprachigen Raum und bei Brendle/Troxell (1949: 80f.) für die USA.

15 Das hier angeführte Beispiel ist bereits bei Füstenberger/González (2002: 14ff.) und noch wesentlich genauer bei Canova (2005: 15ff.) beschrieben worden.

16 So etwa "Spalentor «, ein ehemaliges Stadttor in Basel, welches in die Stadtmauer integriert war. 
Response-Prinzip wird angewendet, das Lied wird im Regelfall zu geselligen Anlässen aufgeführt und ist selten konzertant. Des Weiteren sind üblicherweise eine Refrainzeile und eine Kettenliedstruktur beobachtbar (vgl. Keel 2002: 21ff., Keel 2003: 11ff. und Keel 2009: 213ff.). Die zugrundeliegende Melodie ähnelt stark dem französischen Kinderlied »Ah! Vous Dirai-je, Maman«, ist aber auch aus vielen weiteren Liedern wie »Twinkle, Twinkle, Little Star «, »Baa Baa Black Sheep« oder »ABCDEFG « bekannt. ${ }^{17}$ Obwohl auch Varianten dokumentiert sind, denen andere Melodien zugrunde liegen ${ }^{18}$, geht die Verbreitung einer prädominanten Version innerhalb verschiedener Varianten so weit, dass Keel gar von einer klassischen, in der Midwest-Region geläufigen Schnitzelbank mit 17 Darstellungen spricht. Er macht hierzu auch Textangaben (vgl. Keel 2003: 223f.):

»Ist das nicht eine Schnitzelbank? Ja, das ist eine Schnitzelbank! Ist das nicht ein Kurz und Lang? Ja, das ist ein Kurz und Lang! Ist das nicht ein Hin und Her? Ja, das ist ein Hin und Her! Ist das nicht ein Kreuz und Quer? Ja, das ist ein Kreuz und Quer! Ist das nicht ein Schies Gewehr? Ja, das ist ein Schies Gewehr! Ist das nicht ein Wagen Rad? Ja, das ist ein Wagen Rad! Ist das nicht ein Krum und Grad? Ja, das ist ein Krum und Grad! Ist das nicht ein grosses Glas? Ja, das ist ein grosses Glas! Ist das nicht ein Oxen Blas? Ja, das ist ein Oxen Blas! Ist das nicht ein Hauffen Mist? Ja, das ist ein Hauffen Mist! Ist das nicht ein Schnickel Fritz? Ja, das ist ein Schnickel Fritz! Ist das nicht eine dicke Frau? Ja, das ist eine dicke Frau! Ist das nicht eine fette Sau? Ja, das ist eine fette Sau! Ist das nicht ein langer Mann? Ja, das ist ein langer Mann! Ist das nicht ein Tanenbaum? Ja, das ist ein Tanenbaum! Ist das nicht ein Hochzeit Ring? Ja, das ist ein Hochzeit Ring! Ist das nicht ein gefahrliches Ding? Ja, das ist ein gefahrliches Ding!«

Die Anlage des Liedes erinnert stark an das bereits 1949 von Korson beschriebene Lied Di Lichtputscher, in dem zwar im Pennsylvania-deutschen Dialekt gesungen wird, aber doch auffällige Ähnlichkeiten feststellbar sind: Aus Kurz und Lang wird »Kaz un Lang«, aus Krum und Grad wird »Grum und Grâd«, aus

17 Vgl. für die unterschiedlichen Versionen Fuld (2012: 593f.) und für die Melodiebeispiele Korson (1949: 80) sowie W.A. Mozarts KV 265 (1778).

18 Ein Beispiel für eine andere Melodie, die ebenfalls mit Deutschland assoziiert werden dürfte, ist das von Joseph Haydn komponierte »Gott erhalte Franz den Kaiser « aus dem späten 18. Jh. Die Einbindung in die US-amerikanische Schnitzelbanktradition kann man exemplarisch im Video »German in the US: Schnitzelbank« (Baum 2009) beobachten. 
Wagen Rad wird »Wajjerâd« etc. (vgl. jeweils Korson 1949: 80-82). Auch Begemanns Untersuchungen zum Liedgut der Pennsylvania-Deutschen zeigen zwei Varianten, die abgesehen von der Schreibweise auffällige sprachliche Ähnlichkeiten mit der Version aus Keels Darstellung aufweisen (vgl. Begemann 1973: 148f.):

»Is des net en Schnitzelbank? Jâ des is en Schnitzelbank.

Un des do en Koz und Lang? Ja des is en Kaz und Lang."

Überhaupt scheint hier die Vereinnahmung des Liedes und die Empfindung als zugehörig zum Liedgut der Pennsylvania-Deutschen aus zwei Gründen für die Tradierung bedeutsam. Begemann (1973: 53) weist zum einen darauf hin, dass die im Kollektiv durchgeführten Arbeitsparties wie die »Apple Butter Party« als Umschlagplatz für Liedgut betrachtet werden können, und zum anderen darauf, dass der Schnitzelbank insofern eine Sonderrolle zufällt, als dass das Lied sowohl als Kinderlied als auch in der Erwachsenenunterhaltung funktioniert. Dieser alle Altersgruppen umfassende Anwendungsbereich führt möglicherweise dazu, dass es einem breiten Spektrum an German-Americans aus der Region um Pennsylvania in Erinnerung bleiben konnte, und macht das Lied somit zum potentiellen Kandidaten für fortlaufende Überlieferung (ebd.: 48).

Nun sind die drei europäischen Vorläufer der US-amerikanischen Tradition der Schnitzelbank als Volkslied beschrieben worden, und es steht aus (vgl. Abb. 2), das Schnitzelbank-Sujet in Popsongs zu beschreiben und zu deuten.

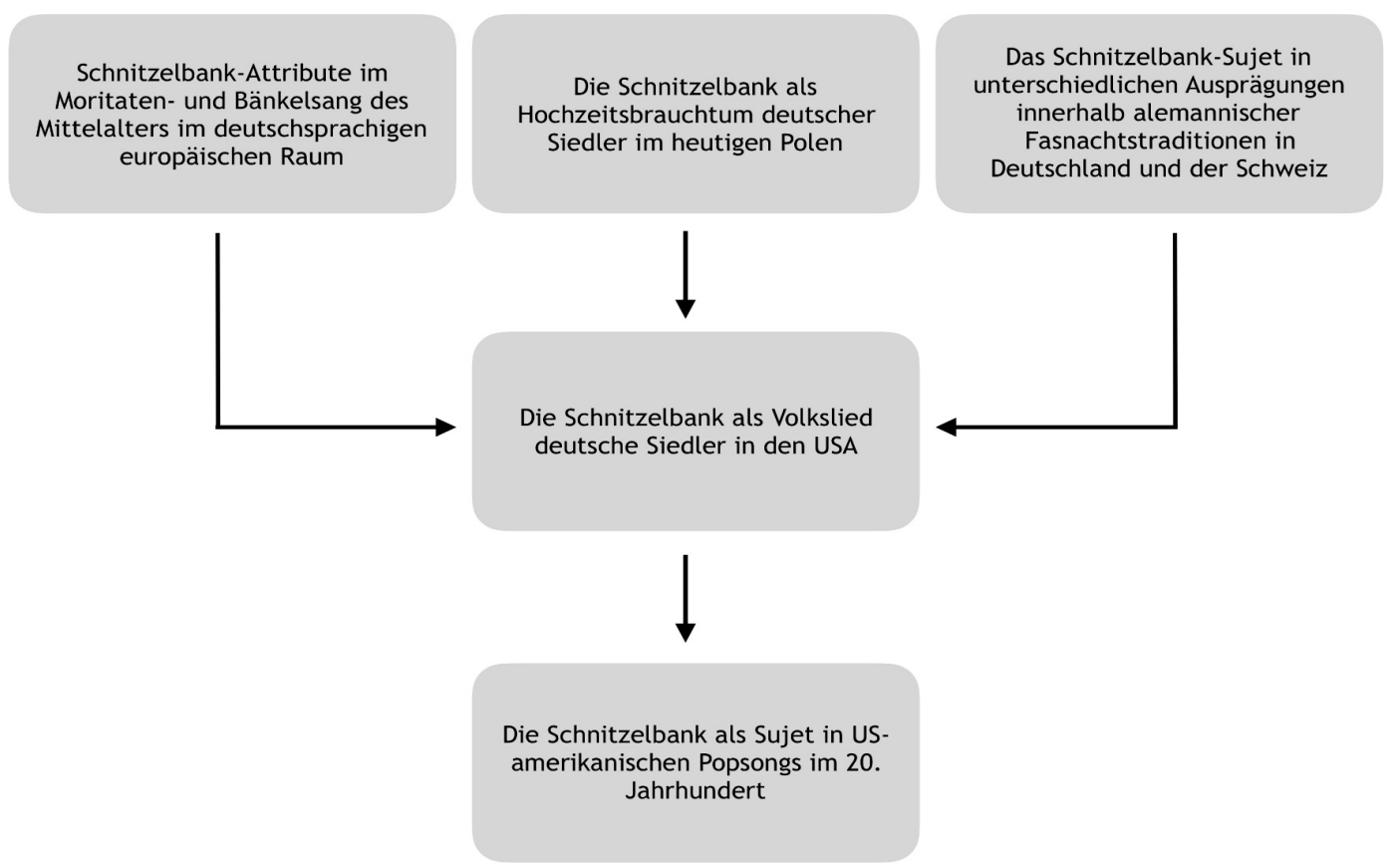

Abb. 2: Die Entwicklung der Schnitzelbank in den USA aus europäischen Traditionen heraus. 


\section{Das Schnitzelbank-Sujet in US-amerikanischen Popsongs}

Schon vor 1945 sind in den USA Aufnahmen der Schnitzelbank erschienen. Hier sind mit der "Schnitzelbank« des Louis Bauer Quartet (1913, Columbia E1315), der »Schnitzelbank« des Manhattan Quartet (1911, Victor C-10326), der Variante »Ei du schöne Schnitzelbank« des Nebe Quartet (1913, Edison Blue Amberol 26082) und der »Schnitzelbank« des Heidelberg Quartette (1922, Gennet 4884-A) mindestens vier Fälle bekannt. Wenn man bedenkt, dass auch in Printmedien schon zu Beginn des 20. Jahrhunderts das Schnitzelbank-Sujet Verwendung findet (vgl. die Abb. 3 sowie die Beschreibung des Schnitzelbank Carnival in Abschnitt 7 dieses Beitrages), wird deutlich, dass die Aufgriffe nach 1945 eine weitere Adaptionsstufe in der Historie der Schnitzelbank in den USA darstellen. Dies ist bemerkenswert, da durchaus ein Bruch zu erwarten gewesen wäre. Die Beschränkung des Datenkorpus für diese Studie auf Songs, die nach 1945 erschienen sind, ist vor allem deshalb von Interesse, da es nicht als Selbstverständlichkeit angesehen werden kann, dass in Zeiten zunehmender Germanophobie nach den beiden Weltkriegen deutschsprachige Begriffe und Musik deutschen Ursprungs kommerziell verwertet werden.

Um das Vorkommen des Schnitzelbank-Sujets in US-amerikanischen Popsongs nach 1945 zu ermitteln, stützt sich diese Studie ausschließlich auf die Einträge der Billboard-Charts, da es auch um die Frage geht, ob es sich nicht möglicherweise um Randerscheinungen handelt, die außerhalb des popkulturellen Mainstreams verortet werden können. Um dies ausschließen zu können, werden die Billboard-Charts hier als Indikator für kommerziellen Erfolg und Massenkompatibilität verwendet. Der Beginn der Erfassung liegt im Jahr 1945 - dem Jahr, in dem die Schnitzelbank erstmals in diesem Kontext in Erscheinung tritt.

Tabelle 1 zeigt die Nennungen des Begriffs Schnitzelbank im BillboardMagazine nach 1945. ${ }^{19}$ Es zeigt sich, dass zwischen dem 12. Mai 1945 und dem

19 Die Darstellung beinhaltet die Ergebnisse der Volltextsuche in allen zur Zeit der Recherche online frei verfügbaren Billboard-Ausgaben über https://books. google.de (Zugriff: 15.4.2018), weiterhin der verfügbaren Ausgaben aus dem Zeitungsarchiv newspapers.com sowie die Ergebnisse von Sichtungen der privaten Sammlung des Autors mit alten Magazinen. Die Liste ist dennoch vermutlich unvollständig. Möglicherweise existieren noch weitere Schnitzelbank-Aufgriffe. Es ist allerdings schwierig dies zu überprüfen, da die Billboard-Ausgaben bislang nur unvollständig digitalisiert worden sind und es zum anderen denkbar ist, dass sich mögliche Sujet-Aufgriffe hinter völlig anderen Titeln verbergen, die hier nicht erfasst wurden. 
FABIAN BADE

\begin{tabular}{|c|c|c|c|c|}
\hline Quelle & $\begin{array}{l}\text { Kategorie } \\
\text { (Kategorisierung im } \\
\text { Magazin) }\end{array}$ & Interpret & $\begin{array}{l}\text { Auffuih- } \\
\text { rungsort }\end{array}$ & $\begin{array}{l}\text { Details zur Auffuihrung / } \\
\text { Aufnahme sowie Songtitel }\end{array}$ \\
\hline $\begin{array}{l}\text { 12.5.1945, } \\
\text { S. } 30\end{array}$ & $\begin{array}{l}\text { Konzertbericht / Night } \\
\text { Clubs-Vaudeville }\end{array}$ & $\begin{array}{l}\text { Sophie } \\
\text { Tucker \& Ted } \\
\text { Shapiro }\end{array}$ & $\begin{array}{l}\text { Chez } \\
\text { Paree, } \\
\text { Chicago, Il }\end{array}$ & $\begin{array}{l}\text { Terrific patriotic parody of } \\
\text { the tune of »Ist Das Nicht } \\
\text { Ein Schnitzelbank«? }\end{array}$ \\
\hline $\begin{array}{l}\text { 13.7.1946, } \\
\text { S. } 41\end{array}$ & $\begin{array}{l}\text { Konzertbericht / Night } \\
\text { Clubs-Vaudeville }\end{array}$ & $\begin{array}{l}\text { Emsee } \\
\text { Cornell } \\
\text { Cooper }\end{array}$ & $\begin{array}{l}\text { Pittsburgh, } \\
\text { PA }\end{array}$ & $\begin{array}{l}\text { Encored with } \\
\text { »Schnitzelbank« }\end{array}$ \\
\hline $\begin{array}{l}\text { 20.7.1946, } \\
\text { S. } 43\end{array}$ & $\begin{array}{l}\text { Konzertbericht / Night } \\
\text { Clubs-Vaudeville }\end{array}$ & Billy Green & $\begin{array}{l}\text { Sherman } \\
\text { Oaks, CA }\end{array}$ & $\begin{array}{l}\text { Billy Green [...] almost } \\
\text { brought the house down } \\
\text { with the old vaude stand- } \\
\text { by, »Schnitzel Bank«. }\end{array}$ \\
\hline $\begin{array}{l}\text { 14.5.1949, } \\
\text { S. } 25\end{array}$ & $\begin{array}{l}\text { Werbeanzeige von } \\
\text { Coral Records }\end{array}$ & $\begin{array}{l}\text { The Ames } \\
\text { Brothers }\end{array}$ & - & $\begin{array}{l}\text { Oh, You Sweet One (the } \\
\text { Schnitzelbank Song) }\end{array}$ \\
\hline $\begin{array}{l}\text { 11.6.1949, } \\
\text { S. } 21\end{array}$ & $\begin{array}{l}\text { Werbeanzeige von } \\
\text { Decca Records }\end{array}$ & $\begin{array}{l}\text { Andrew } \\
\text { Sisters }\end{array}$ & - & $\begin{array}{l}\text { Oh, You Sweet One (the } \\
\text { Schnitzelbank Song }\end{array}$ \\
\hline $\begin{array}{l}\text { 9.7.1949, } \\
\text { S. } 150\end{array}$ & $\begin{array}{l}\text { Auflistung Neuerschei- } \\
\text { nungen: International }\end{array}$ & $\begin{array}{l}\text { The Weidler } \\
\text { Brothers }\end{array}$ & - & The Schnitzelbank Polka \\
\hline $\begin{array}{l}\text { 30.7.1949, } \\
\text { S. } 45\end{array}$ & $\begin{array}{l}\text { Werbeanzeige von } \\
\text { RCA/Victor Records }\end{array}$ & Joe Biviano & - & Schnitzel Bank \\
\hline $\begin{array}{l}13.8 .1949 \\
\text { S. } 111\end{array}$ & $\begin{array}{l}\text { Bewertungen durch } \\
\text { Disc-Jockeys }\end{array}$ & Joe Biviano & - & $\begin{array}{l}\text { Another popular oldie gets } \\
\text { a spirited, good-humored } \\
\text { going-over from the fine } \\
\text { vocal group and lively band } \\
\text { [...]. }\end{array}$ \\
\hline $\begin{array}{l}27.8 .1949, \\
\text { S. } 103\end{array}$ & Konzertbericht & $\begin{array}{l}\text { The Weidler } \\
\text { Brothers }\end{array}$ & $\begin{array}{l}\text { Los } \\
\text { Angeles, CA }\end{array}$ & $\begin{array}{l}\text { [...] had George, Walter } \\
\text { and Warner Weidler [...] as } \\
\text { recent guests. They were } \\
\text { pitching for their new } \\
\text { "Schnitzelbank Polka«... }\end{array}$ \\
\hline $\begin{array}{l}1.10 .1949, \\
\text { S. } 21\end{array}$ & $\begin{array}{l}\text { Werbeanzeige von } \\
\text { Vokalion Records }\end{array}$ & $\begin{array}{l}\text { Leighton } \\
\text { Noble }\end{array}$ & - & $\begin{array}{l}\text { Oh, You Sweet One (the } \\
\text { Schnitzelbank Song) }\end{array}$ \\
\hline $\begin{array}{l}22.10 .1949, \\
\text { S. } 79\end{array}$ & $\begin{array}{l}\text { Werbeanzeige von RCA } \\
\text { Victor Records }\end{array}$ & Joe Biviano & - & Schnitzel Bank \\
\hline $\begin{array}{l}31.3 .1951 \\
\text { S. } 31\end{array}$ & $\begin{array}{l}\text { Werbeanzeige von RCA } \\
\text { Victor Records }\end{array}$ & $\begin{array}{l}\text { Lawrence } \\
\text { Duchow \& his } \\
\text { RCA-Victor } \\
\text { Recording } \\
\text { Orchestra }\end{array}$ & - & $\begin{array}{l}\text { Oh You Sweet One (The } \\
\text { New Schnitzelbank Song) }\end{array}$ \\
\hline $\begin{array}{l}25.4 .1953, \\
\text { S. } 99\end{array}$ & $\begin{array}{l}\text { Kleinanzeige in der } \\
\text { Rubrik Miscellaneous }\end{array}$ & $\begin{array}{l}\text { kein spezifi- } \\
\text { scher } \\
\text { Interpret }\end{array}$ & $\begin{array}{l}\text { Hoboken, } \\
\text { NJ }\end{array}$ & $\begin{array}{l}{[\ldots] \text { walkaround }} \\
\text { equipment allows taxless } \\
\text { music and Schnitzelbank } \\
\text { acts }[. . .] \text {. }\end{array}$ \\
\hline $\begin{array}{l}\text { 12.5.1956, } \\
\text { S. } 100\end{array}$ & $\begin{array}{l}\text { Reviews of New Pop } \\
\text { Records }\end{array}$ & Hal Dickinson & - & $\begin{array}{l}\text { Using the »Schnitzelbank } \\
\text { Song « and »O du lieber } \\
\text { Augustin« as the basis of } \\
\text { his material. The German } \\
\text { Band, Dickinson lampoons } \\
\text { the oompah bands beloved } \\
\text { by the Germans. }\end{array}$ \\
\hline $\begin{array}{l}11.8 .1956, \\
\text { S. } 60\end{array}$ & $\begin{array}{l}\text { Kleinanzeige RCA } \\
\text { Victor Records }\end{array}$ & $\begin{array}{l}\text { Six Fat } \\
\text { Dutchmen }\end{array}$ & - & Schnitzelbank Polka \\
\hline
\end{tabular}

Tab. 1: Schnitzelbank-Einträge im Billboard-Magazine nach 1945. 
17. Februar 1962 mindestens zwölf Interpreten oder Gruppen sich der Schnitzelbank auf verschiedene Weisen widmeten: Das Sujet ist bei Sophie Tucker \& Ted Shapiro, Emsee Cornell Cooper, Billy Green sowie den Weidler Brothers als Live-Performance dokumentiert, Schallplattenaufnahmen sind von den Ames Brothers, den Andrew Sisters, den Weidler Brothers, Joe Biviano, Leighton Noble, Lawrence Duchow, Hal Dickinson, den Six Fat Dutchmen sowie Rudy Vallee erschienen.

Bemerkenswert ist die Tatsache, dass die Aufführungsorte (an zwei Orten in Kalifornien und je einmal in Illinois und Pennsylvania) recht weit auseinanderliegen. Aufgrund der Datenarmut und anhand der dokumentierten Darbietungen lässt sich allerdings vorerst nicht ausschließen, dass es sich möglicherweise doch um ein regionales Phänomen handelt, was zumindest denkbar ist, wenn man die Migrationsbewegungen aus Deutschland in die USA betrachtet und bedenkt, dass ein Ungleichgewicht bzgl. der Verteilung von Siedlungen deutschstämmiger US-Bürger in den einzelnen Bundesstaaten besteht (vgl. New York Times Immigration Explorer 2009).

Die in Tab. 1 aufgelisteten Aufnahmen entstanden in einem Zeitraum von etwa dreizehn Jahren zwischen dem 14. Mai 1949 (Ames Brothers) und dem 17. Februar 1962 (Rudy Vallee). Nicht nur der ungewöhnlich lange Zeitraum, in dem die Schnitzelbank immer wieder Teil der US-Popkultur war, ist hier auffällig, sondern auch die Diversität der Interpretationen von eher seriösen Aufnahmen (die Andrew Sisters-Version aus den 1940er Jahren) bis hin zur bierseligen Trinkliedatmosphäre, die die Interpretation der Six Fat Dutchmen vermittelt. ${ }^{20}$

Die hier beschriebenen Aufnahmen und Aufführungen sind aber nur die sprichwörtliche Spitze des Eisberges. Weitere und vor allem umfangreichere Korpus-Analysen wären vonnöten, um ein genaueres Bild der SchnitzelbankRezeption in den USA nachzuzeichnen. Der hier gegebene Rahmen ermöglicht es lediglich, einige weitere Schlaglichter zu werfen. Wie Tab. 1 zeigt, findet sich die Schnitzelbank in 25\% der Einträge auch unter dem Titel »Oh, You Sweet One « wieder, partiell mit dem Zusatz »the Schnitzelbank Song « oder »The New Schnitzelbank Song«. Tabelle 2 listet die Einträge mit dem Titel »Oh, You Sweet One« auf. ${ }^{21}$ Die 35 Einträge stammen aus dem Zeitraum 1949

20 Vgl. exemplarisch die RCA Victor-Aufnahme der Schnitzelbank vom Album Six Merry Polkas der Six Fat Dutchmen aus dem Jahr 1957 unter https://www. youtube.com/watch?v=G8NfOdNDuy0 ab Minute 8:00.

21 Es wurden folgende Begriffe auf Übereinstimmungen mit den Billboard-Charts überprüft: Schnitzelbank, Hobelbank, Snydersbank und Lichtputzschere. Es ist denkbar, dass aufgrund sich verändernder Dialekte oder Übertragungsfehler (vgl. exemplarisch Korson 1949: 80f.) noch weitere Varianten existieren. 


\begin{tabular}{|c|c|c|c|}
\hline Quelle & $\begin{array}{l}\text { Kategorie } \\
\text { (Kategorisierung im } \\
\text { Magazin) }\end{array}$ & Interpret & $\begin{array}{l}\text { Details zur Aufführung / Aufnahme } \\
\text { sowie Songtitel }\end{array}$ \\
\hline $\begin{array}{l}\text { 7.5.1949, } \\
\text { S. } 25\end{array}$ & $\begin{array}{l}\text { Anzeige und Bestell- } \\
\text { Liste (Coral-Records) }\end{array}$ & $\begin{array}{l}\text { vermutl. Ames } \\
\text { Brothers }\end{array}$ & $\begin{array}{l}\text { Oh, You Sweet One (The Schnitzelbank } \\
\text { Song) }\end{array}$ \\
\hline $\begin{array}{l}14.5 .1949, \\
\text { S. } 36\end{array}$ & $\begin{array}{l}\text { Vorankündigungen von } \\
\text { Veröffentlichungen }\end{array}$ & Ames Brothers & Oh, You Sweet One \\
\hline $\begin{array}{l}21.5 .1949 \\
\text { S. } 116\end{array}$ & $\begin{array}{l}\text { Bewertungen durch } \\
\text { Disc-Jockeys }\end{array}$ & Ames Brothers & $\begin{array}{l}\text { Oh, You Sweet One. Lyric set to the } \\
\text { »Schitzelbank Song « (!) makes for a } \\
\text { downright infectious etching [...]. }\end{array}$ \\
\hline $\begin{array}{l}18.6 .1949 \\
\text { S. } 21\end{array}$ & $\begin{array}{l}\text { Anzeige (Decca- } \\
\text { Records) }\end{array}$ & $\begin{array}{l}\text { Andrew Sisters } \\
\text { and Russ } \\
\text { Morgan and } \\
\text { his Orchestra }\end{array}$ & Oh, You Sweet One \\
\hline $\begin{array}{l}25.6 .1949 \\
\text { S. } 36\end{array}$ & $\begin{array}{l}\text { Konzertbericht / On } \\
\text { The Stand }\end{array}$ & Russ Morgan & $\begin{array}{l}\text { Konzert in Hollywood, CA. His current } \\
\text { release »Oh, You Sweet One « [...] is } \\
\text { expected to be still another strong } \\
\text { contender for platter coin. }\end{array}$ \\
\hline $\begin{array}{l}9.7 .1949 \\
\text { S. } 19\end{array}$ & $\begin{array}{l}\text { Anzeige (Decca- } \\
\text { Records) }\end{array}$ & $\begin{array}{l}\text { Andrew Sisters } \\
\text { and Russ } \\
\text { Morgan }\end{array}$ & $\begin{array}{l}\text { Oh, You Sweet One (The Schnitzelbank } \\
\text { Song) }\end{array}$ \\
\hline $\begin{array}{l}\text { 16.7.1949, } \\
\text { S. } 39\end{array}$ & $\begin{array}{l}\text { Vorankündigungen von } \\
\text { Veröffentlichungen }\end{array}$ & $\begin{array}{l}\text { Andrew Sisters } \\
\text { and Russ } \\
\text { Morgan }\end{array}$ & Oh, You Sweet One \\
\hline $\begin{array}{l}\text { 16.7.1949, } \\
\text { S. } 39\end{array}$ & $\begin{array}{l}\text { Vorankündigungen von } \\
\text { Veröffentlichungen }\end{array}$ & \begin{tabular}{|l|} 
Leighton \\
Noble
\end{tabular} & Oh, You Sweet One \\
\hline $\begin{array}{l}30.7 .1949, \\
\text { S. } 43\end{array}$ & $\begin{array}{l}\text { Anzeige (Decca- } \\
\text { Records) }\end{array}$ & $\begin{array}{l}\text { Andrew Sisters } \\
\text { and Russ } \\
\text { Morgan }\end{array}$ & $\begin{array}{l}\text { Oh, You Sweet One (The Schnitzelbank } \\
\text { Song) }\end{array}$ \\
\hline $\begin{array}{l}\text { 13.8.1949, } \\
\text { S. } 35\end{array}$ & $\begin{array}{l}\text { Bewertungen durch } \\
\text { Disc-Jockeys }\end{array}$ & $\begin{array}{l}\text { Leighton } \\
\text { Noble }\end{array}$ & $\begin{array}{l}\text { Oh, You Sweet One. The Schnitzelbank } \\
\text { song gets like treatment. }\end{array}$ \\
\hline $\begin{array}{l}\text { 3.9.1949, } \\
\text { S. } 17\end{array}$ & $\begin{array}{l}\text { Anzeige (Decca- } \\
\text { Records) }\end{array}$ & $\begin{array}{l}\text { Andrew Sisters } \\
\text { and Russ } \\
\text { Morgan }\end{array}$ & $\begin{array}{l}\text { Oh, You Sweet One (The Schnitzelbank } \\
\text { Song) }\end{array}$ \\
\hline $\begin{array}{l}\text { 10.9.1949, } \\
\text { S. } 21\end{array}$ & $\begin{array}{l}\text { Anzeige (Vocalion } \\
\text { Records) }\end{array}$ & $\begin{array}{l}\text { Leighton } \\
\text { Noble }\end{array}$ & $\begin{array}{l}\text { Oh, You Sweet One (The Schnitzelbank } \\
\text { Song) }\end{array}$ \\
\hline $\begin{array}{l}1.10 .1949, \\
\text { S. } 21\end{array}$ & $\begin{array}{l}\text { Anzeige (Vocalion } \\
\text { Records) }\end{array}$ & $\begin{array}{l}\text { Leighton } \\
\text { Noble }\end{array}$ & $\begin{array}{l}\text { Oh, You Sweet One (The Schnitzelbank } \\
\text { Song) }\end{array}$ \\
\hline $\begin{array}{l}\text { 14.1.1950, } \\
\text { S. } 15\end{array}$ & $\begin{array}{l}\text { Billboard- } \\
\text { Jahresbestenliste }\end{array}$ & Andrew Sisters & Oh, You Sweet One \\
\hline $\begin{array}{l}\text { 20.5.1950, } \\
\text { S. } 22\end{array}$ & $\begin{array}{l}\text { Hitliste: Platz } 17 \text { in der } \\
\text { Kategorie England's Top } \\
\text { Twenty }\end{array}$ & - & Oh, You Sweet One \\
\hline $\begin{array}{l}27.5 .1950 \\
\text { S. } 22\end{array}$ & $\begin{array}{l}\text { Hitliste: Platz } 7 \text { in der } \\
\text { Kategorie England's Top } \\
\text { Twenty }\end{array}$ & - & Oh, You Sweet One \\
\hline $\begin{array}{l}3.6 .1950, \\
\text { S. } 22\end{array}$ & $\begin{array}{l}\text { Hitliste: Platz } 5 \text { in der } \\
\text { Kategorie England's Top } \\
\text { Twenty }\end{array}$ & - & Oh, You Sweet One \\
\hline $\begin{array}{l}\text { 10.6.1950, } \\
\text { S. } 22\end{array}$ & $\begin{array}{l}\text { Hitliste: Platz } 5 \text { in der } \\
\text { Kategorie England's Top } \\
\text { Twenty }\end{array}$ & - & Oh, You Sweet One \\
\hline
\end{tabular}

Tab. 2: »Oh, You Sweet One«-Einträge im Billboard-Magazine nach 1945. 
bis 1956 und fügen der oben genannten Aufzählung von zwölf Interpreten respektive Gruppen mit Thurl Ravenscroft noch einen weiteren hinzu.

Zusammenfassend betrachtet deuten die oben genannten Anzeigen, Konzertberichte, Vorankündigungen, Bewertungen in verschiedenen Größen sowie Hitparaden aus dem im Rahmen der Volltextsuche ermittelten Datenkorpus auf die Diversität der Kontexte hin, in denen die Schnitzelbank in den USA in diesem Zeitraum vorkam. Es wird sichtbar, dass die Schnitzelbank auch als Popsong in weit mehr als nur Einzelfällen Eingang in die Popkultur fand. Ein Beispiel für einen in den tabellarischen Darstellungen nicht enthaltenen Song ist Bill Haleys »Rockin' Rollin' Schnitzlebank« vom 1956 veröffentlichen Decca-Album Rockin' Around The World. Obwohl diese Version von einem der die 1950er Jahre musikalisch entscheidend mitprägenden Interpreten stammt, findet sie im Billboard-Magazine nicht weiter Erwähnung. Ein Grund hierfür mag darin liegen, dass der Titel in den Vereinigten Staaten nie als Single ausgekoppelt wurde. ${ }^{22}$ Dennoch deutet gerade der Sujet-Aufgriff durch so unterschiedliche Interpreten wie Rudy Vallee, die Andrew Sisters, Bill Haley oder die Six Fat Dutchmen darauf hin, dass die Schnitzelbank nicht nur an bestimmten regionalen Hotspots, sondern landesweit bekannt gewesen sein muss. Zwar kommen einige Interpreten aus Regionen mit höherer Dichte deutscher Einwanderer oder US-Amerikanern mit deutschen Wurzeln ${ }^{23}$, jedoch stammen ebenso viele aus ganz anderen Regionen. Vielmehr noch ist hier die Verteilung auffällig: Mit den Ames Brothers aus Massachusetts, George Weidler aus Los Angeles, Thurl Ravenscroft aus Nebraska oder Bill Haley aus Michigan stammen die Interpreten aus unterschiedlichsten Regionen der Vereinigten Staaten. Die popkulturelle Relevanz national berühmter Interpreten und Künstler wie den Andrew Sisters und Bill Haley allein verdeutlicht noch einmal die mediale Reichweite, von der die SchnitzelbankBekanntheit sicherlich profitiert haben dürfte.

Nachdem nun die der Schnitzelbank zugrundeliegenden Traditionen aus dem zentraleuropäischen Raum bekannt sind und der Schnitzelbank-Song in den USA und das Auftreten des Schnitzelbank-Sujets in US-amerikanischen Popsongs systematisch erfasst und beschrieben wurden (vgl. Tab. 1 u. 2), soll

22 Vgl. https://www.discogs.com/de/Bill-Haley-And-His-Comets-Rockin-Rollin-Schnit zelbank/release/7627991, Zugriff: 15.4.2018.

23 Russ Morgen kam aus Pennsylvania, die Six Fat Dutchmen und die Andrew Sisters wurden allesamt in Minnesota geboren. Beide Bundesstaaten haben laut Census einen überdurchschnittlich hohen Anteil an US-Amerikanern mit deutschen Vorfahren. Dieser Wert liegt im Durchschnitt bei 14,9\%, beträgt aber in einigen Counties in Minnesota bis zu 40\% (vgl. https://www.census.giv/library/ visualizations/2016/comm/german-roots.html, Zugriff: 15.4.2018). 
abschließend eine tabellarische Darstellung den Überblick im Sinne einer Zusammenfassung vereinfachen:

\begin{tabular}{|c|c|c|c|c|c|c|c|c|}
\hline $\begin{array}{c}\text { Merkmale } \\
\text { (v.l.n.r.) }\end{array}$ & Sprache & $\begin{array}{l}\text { Ketten- } \\
\text { lied- } \\
\text { Struktur }\end{array}$ & $\begin{array}{c}\text { Freie } \\
\text { Versform }\end{array}$ & $\begin{array}{c}\text { Refrain- } \\
\text { zeile }\end{array}$ & Bildtafel & $\begin{array}{l}\text { Call- \& } \\
\text { Response } \\
\text {-Prinzip }\end{array}$ & $\begin{array}{l}\text { Humo- } \\
\text { reske } \\
\text { Inhalte }\end{array}$ & $\begin{array}{l}\text { Beleh- } \\
\text { rung \& } \\
\text { Moral }\end{array}$ \\
\hline $\begin{array}{l}\text { Moritaten- } \\
\text { und Bänkel- } \\
\text { sang }\end{array}$ & $\begin{array}{l}\text { Versch. } \\
\text { Dialekte }\end{array}$ & Nein & $\begin{array}{l}\text { Nicht } \\
\text { zwingend }\end{array}$ & Nein & $\mathrm{Ja}$ & Nein & $\mathrm{Ja}$ & Häufig \\
\hline $\begin{array}{l}\text { Hochzeits- } \\
\text { Brauchtum } \\
\text { deutscher } \\
\text { Siedler in } \\
\text { Polen }\end{array}$ & $\begin{array}{l}\text { Dt. bzw. } \\
\text { Dialekt }\end{array}$ & $\mathrm{Ja}$ & Nein & Unklar & $\begin{array}{l}\text { Entsteht } \\
\text { während } \\
\text { Auffüh- } \\
\text { rung }\end{array}$ & $\mathrm{Ja}$ & $\mathrm{Ja}$ & Nein \\
\hline $\begin{array}{l}\text { Fastnachts- } \\
\text { Brauchtum in } \\
\text { Basel }\end{array}$ & $\begin{array}{l}\text { Basel-dt. } \\
\text { (Nieder- } \\
\text { aleman- } \\
\text { nisch) }\end{array}$ & Nein & $\mathrm{Ja}$ & $\begin{array}{l}\text { Teil- } \\
\text { weise }\end{array}$ & $\mathrm{Ja}$ & Nein & $\mathrm{Ja}$ & $\mathrm{Ja}$ \\
\hline $\begin{array}{l}\text { Fastnachts- } \\
\text { Brauchtum in } \\
\text { Ellwangen }\end{array}$ & $\begin{array}{l}\text { Schwä- } \\
\text { bisch } \\
\text { (Aleman- } \\
\text { nisch) }\end{array}$ & Nein & $\mathrm{Ja}$ & Unklar & Unklar & Nein & $\mathrm{Ja}$ & $\mathrm{Ja}$ \\
\hline $\begin{array}{l}\text { Schnitzel- } \\
\text { bank-Song in } \\
\text { den USA }\end{array}$ & $\begin{array}{l}\text { Dt. mit } \\
\text { Überlie- } \\
\text { ferungs- } \\
\text { fehlern }\end{array}$ & $\mathrm{Ja}$ & Nein & $\mathrm{Ja}$ & $\mathrm{Ja}$ & $\mathrm{Ja}$ & $\mathrm{Ja}$ & Nein \\
\hline $\begin{array}{l}\text { Schnitzel- } \\
\text { bank-Sujet in } \\
\text { US-Popsongs }\end{array}$ & $\begin{array}{l}\text { Engl. u. } \\
\text { verein- } \\
\text { zelt dt. }\end{array}$ & $\begin{array}{l}\text { Noch er- } \\
\text { kennbar, } \\
\text { jedoch } \\
\text { stark } \\
\text { verkürzt }\end{array}$ & Nein & \begin{tabular}{|l|} 
Ja, aber \\
i.d.R. \\
abgeän- \\
dert
\end{tabular} & Nein & Teilweise & $\mathrm{Ja}$ & Nein \\
\hline
\end{tabular}

Tab. 3: Merkmale der Schnitzelbank-Traditionen und ihrer Vorläufer im Vergleich.

\section{Das Schnitzelbank-Sujet in der US- amerikanischen Popkultur}

Keel argumentierte bereits, dass die Schnitzelbank angesichts der hohen Frequenz, mit der sie in der US-amerikanischen Popkultur auftaucht, offenbar ins kulturelle Gedächtnis der USA eingebrannt sei (vgl. Keel 2003: 221). Zu den prominentesten Beispielen können die Verwendungen der Schnitzelbank in Filmen, TV-Serien und Literatur gezählt werden. Keel verweist u.a. auf die Filme Horsefeathers der Marx Brothers (1932), I'll Never Heil Again der Three Stooges (1941) und Stalag 17 von Billy Wilder (1953), auf Serien wie I Love Lucy aus den 1950er Jahren oder Steven Spielbergs Animaniacs aus den 1990er 
Jahren sowie auf Literatur von Betty Smith und Katherine Anne Porter. Die Tatsache, dass das Sujet nicht nur als Volkslied in mannigfaltigen Ausprägungen oder als Popsong in verschiedenen Abwandlungen, sondern auch in diesen Filmen und Fernsehserien sowie darüber hinaus nicht selten in Printwerbungen ortsunabhängig und über einen längeren Zeitraum präsent ist, macht umso deutlicher, dass auch die der Schnitzelbank zugrunde liegenden Formprinzipien wie die Kettenliedstruktur, das Call and Response-Prinzip und die Melodie in den USA verbreitet gewesen sein müssen. Denn die Texte sind hier oft stark verändert oder meiden den Begriff Schnitzelbank gar. Zudem sind nicht immer alle genannten Formprinzipien gleichermaßen sichtbar, dies ist aber für die Klassifizierung als Schnitzelbank auch gar nicht nötig: Seemann (1959) erklärt, dass es zum Typ des »Schwellliedes « gehöre, dass »vielfach improvisiert « werde und sich je »nach Versmaß und Melodie die unterschiedlichsten Gestalten entwickeln« können. Abschließend sollen einige Beispiele aus verschiedenen Orten innerhalb der USA dies noch verdeutlichen:

Bewerbung von Produkten oder Gastronomie in der Printwerbung 1951 wirbt die Beverage Sales Co. in Maryland für Old Reading Beer, ein traditionelles Bier der Pennsylvania-Deutschen. Das Getränk selbst ist allerdings innerhalb einer ganzseitigen Werbeanzeige an exponierter Stelle in der Daily Mail aus Maryland vom 27.3.1951 (S. 3) nicht im Zentrum der Anzeige zu finden, der Fokus liegt vielmehr auf der Überschrift »Let's sing the Schnitzelbank Song «. Darunter folgen die Zeilen »Ist das nicht Old Reading Beer? Ya das ist Old Reading Beer. Ist das nicht die best bei mir? Ya das ist die best bei mir « und der übliche Chorus »Ei du schoene «. ${ }^{24}$ Etwa 2760 Meilen westlich von Maryland wirbt das Mountain Home Inn im Daily Independent Journal aus San Rafael in Kalifornien am 23.7.1954 mit den Zeilen: »Ist das nicht ein Schnitzelbank? Nein es ist das bergheim schenke. «25

24 Vgl. ein Digitalisat der Anzeige unter https://www.newspapers.com/image/ 27972791, Zugriff: 15.4.2018.

25 Vgl. ein Digitalisat der Anzeige unter https://www.newspapers.com/image/ 70348786, Zugriff: 15.4.2018. 
Und in Pennsylvania, einer ersten Heimat der frühen German-Americans, die im späten 17. Jahrhundert den Bundesstaat besiedelten, fragt sich ein Autor 1909 in der Reading Times in der Anzeige »Schnitzelbank. Ei du Schoene, Ei du Schoene, Ei du Schoene Schnitzelbank« gar »Who has not heard of the Schnitzelbank? «26

\section{S C H N I T Z E L B A N K}

“Ei Du Schoene, Ei Du Schoene, Ei Du Schoene, Schnitze'bank!”

Who has not heard of the Schnitzelbank? Those who attended the Board of Trade and Merchants' Association pienic at Heller's Park hrard this funny German song; it was the hit of the day. More fun than a circus-mirth and melody. the kind that is dear to the heart of all Germans. On the order of "The House That Jack Built." It is a mirthproducer from start to finish. With several copies of this song. and one of the charts, you have provided fine entertainment for a social gathering.

A good laugh is the best of tonies, and those who cannot enjoy the Schnitzelbank are, indeed, dead ones.

Try the Schnitzel bank tonic-get a sehnitzelbank outfit, so that when your friends call you can have a "barrel of fun:"

Schnitzel bank Comic Song, words and music, piarı copy .................................

Schnitzelbank Chart, $28 \times 39$ inches, printed on cloth

35c Now on sale a cour store.

\section{J. GEORGe hINTZ, 756 Penn Street}

The Big Post Card and Stationery store.

Abb. 3: Schnitzelbank-Anzeige in der Reading Times vom 31.8.1909, https: / www. news papers.com/image/44821265, Zugriff: 15.4.2018.

\section{Benennungen ganzer Veranstaltungen nach der Schnitzelbank}

In Nashville wird im Herbst des Jahres 1903 ein dreizehntägiger Schnitzelbank Carnival abgehalten. Auffällig ist nicht nur die Bewerbung der Veranstaltung selbst, sondern auch die Tatsache, dass Veränderungen im Management der Veranstaltung der Öffentlichkeit kommuniziert werden. Von öffentlichem Interesse in der Region Nashville im Jahr 1903 kann also ausgegangen werden. ${ }^{27}$

26 In der Reading Times vom 31.8.1909 ist das Sujet allgegenwärtig: Der »hit of the day» verspreche »more fun than a circus«. Ein »Schnitzelbank-Tonic «, »Schnitzelbank-Outfit«, Notendruck und Memorabilia - nahezu alle angebotenen Leistungen scheinen hier die Schnitzelbank im Namen zu tragen. Vgl. ein Digitalisat unter https://www.newspapers.com/image/44821265, Zugriff: 15.4.2018.

27 Vgl. hierzu zwei Digitalisate aus dem Tennessean aus den Monaten September und Oktober 1903 unter https://www.newspapers.com/image/118980034 sowie unter https://www.newspapers.com/image/118976925, Zugriff: 15.4.2018. 
Leserbriefe

Im August 1974 schreibt ein Louis Fagon einen Brief an die Herausgeber des Kingston Daily Freeman in New York State, in dem es um die Frage nach der Rechtsgrundlage einer Anhebung der Kraftstoffkosten geht: »Perhaps we should join in the chorus of the Schnitzelbank [...] Song: Ist das nicht ein rate increase? Ja, das ist ein rate increase. ${ }^{28}$

\section{Wahlkampagnen}

1948 berichtet die New York Times, dass bei einer Wahlveranstaltung im Kontext der bevorstehenden Präsidentschaftswahlen das Publikum für den Kandidaten Henry Wallace eine $\gg$ Wallace Schnitzelbank « singen wird. ${ }^{29}$

Jährliche Großveranstaltungen

Ein aktuelles Beispiel ist das »Strassenfest « in Jasper im US-Bundesstaat Indiana, wo jährlich ein »Wettlauf « (5K Road Race) stattfindet. Auf dem T-Shirt zur Veranstaltungsauflage im Jahr 2017 bilden dem Anlass angepasste Reimpaare die Grundlage für »The Wettlauf Running Song«: »5K Run - Starting Gun, Carb Load - Hit The Road, Outdoor John - Jasperbaum, Running Shoe - Shorts Too, Gatorade Cup - Speed It Up, Sauerkraut - Jasper Route, Run Or Walk - Timers Clock, Finish Line - Trophy Time. « Auch hier deuten die Bilder auf eine Verbindung zur Schnitzelbank-Tradition. ${ }^{30}$

\section{Fazit}

Die hier dargestellten Musik- und Textbeispiele aus verschiedenen Landesteilen aus den Jahren zwischen 1903 und 2017 zeigen die mannigfaltige Ausprägung der Schnitzelbank in den USA. Ebenso wie dem Schnitzelbank-Sujet ursprünglich im europäischen Raum verschiedene Funktionen innewohnten resp. zugeschrieben wurden, so besteht im 20. Jahrhundert zwar nicht mehr der gleiche Facettenreichtum wie bei den europäischen Vorläufern, jedoch scheint das Sujet immer noch geeignet, heterogene Anwendungsbereiche abzudecken. So werden Restaurants, Lauf- und Karnevalsveranstaltungen und auch Bier beworben. Bei Wahlkampfveranstaltungen und Leserbriefen findet sich die Schnitzelbank ebenso wieder wie in den von Keel besprochenen Filmen, Serien und Büchern. Die Abwandlung des Schnitzelbank-Songs in den

28 Vgl. den Leserbrief in voller Länge unter https://www.newspapers.com/image/ 86089145, Zugriff am 15.4.2018.

29 Vgl. New York Times vom 24.10.1948, S. 51.

30 Vgl. https://jasperstrassenfest.org/wp-content/uploads/2017/06/2017-WettlaufBrochure.pdf sowie http://cargocollective.com/christianpaeth/Wettlauf-TShirt, Zugriff: 15.4.2018. 
USA für diese differierenden Anlässe kann nur dann sinnvoll sein, wenn die Kommunikation dieses Sujets von den Rezipienten verstanden wird. Das bedeutet, dass die Schnitzelbank samt Text und Formprinzipien unabhängig von regionalen Hotspots deutsch-amerikanischer Kultur bekannt gewesen sein muss. Andernfalls ließen sich die verschiedenen Aufgriffe nur schwer erklären. Keel nennt die Historie der Schnitzelbank mit einem ironischen Blick auf die Kettenliedstruktur »truly a never-ending one « (vgl. Keel 2003: 238). Ob sie wirklich nie endet, sei dahingestellt, dennoch deuten die ausgewerteten Daten darauf hin, dass das Schnitzelbank-Sujet in den USA über das 20. Jahrhundert hinweg durchgehend bekannt gewesen sein muss. Interessant ist, dass genau dies bislang kaum untersucht wurde und dieses Stück deutschamerikanischer Kultur diesseits des Atlantiks so unbekannt ist. Dieser Beitrag kann nun als Ausgangspunkt für Rezeptionsstudien dienen, wobei vor allem interessant sein dürfte herauszufinden, wer die Schnitzelbank wann und wie rezipiert hat respektive wie es um die Rezeption nach der Jahrtausendwende bestellt ist. Hierbei könnten die systematisierten Daten hilfreich und ein Startpunkt für weitere Überlegungen sein.

\section{Literatur}

Anonymous (1948). »Wallace Prepares for Last Speeches«. In: New York Times vom 24.10., S. 51, https://timesmachine.nytimes.com/timesmachine/1948/10/24/ 110057433.html, Zugriff: 15.4.2018.

Baum, Stephan (2009). "German in the US: Schnitzelbank«, https://www.youtube. com/watch?v=c-ygUMCdaJk, Zugriff: 15.4.2018.

Begemann, Renate (1973). Die Lieder der Pennsylvaniadeutschen in ihrem sozialen Kontext. Eine Analyse der Brendelschen Sammlung 1935-1950. Dissertation: Universität Marburg [im Archiv des ZPKM Freiburg: V 1/843].

Brendle, Thomas R. / Troxell, William S. (1949). »Pennsylvania German Songs. «In: Pennsylvania Songs \& Legends. Hg. v. George Korson. Baltimore: Johns Hopkins University Press.

Canova, Ruth (2005). Jo, das isch e Schnitzelbangg. Die Geschichte des Basler Schnitzelbankwesens. Basel: Spalentor.

Fuld, James J. (2012). The Book of World-Famous Music: Classical, Popular, and Folk. Mineola: Dover.

Fürstenberger, Marcus / González, Emelyn (2002). Ei du scheene... Das 20. Jahrhundert im Spiegel der Basler Schnitzelbänke. Basel: Schwabe \& Co.

Gerny, Daniel (2018). »Trump, Erdogan und Billag: Die Basler Fasnacht 2018 ist politisch«. In: Neue Zürcher Zeitung vom 20.2., https://www.nzz.ch/panorama/ trump-erdogan-und-billag-die-basler-fasnacht-2018-ist-politisch-ld.1358881, Zugriff: 1.7.2018.

Hadamer, Armin W. (2001). »German Melodies in American Songs. Beispiele populärer Revival-Lieder der USA mit Wurzeln im deutschsprachigen Kulturraum.« In: 
Populäre Musik im kulturwissenschaftlichen Diskurs II. Hg. v. Thomas Phleps (= Beiträge zur Popularmusikforschung 27/28). Karben: CODA, S. 119-135.

Hadamer, Armin W. (2008). Mimetischer Zauber. Die englischsprachige Rezeption deutscher Lieder in den USA 1830-1880. Münster: Waxmann.

Joseph, Eugen (1885). Konrads von Würzburg Klage der Kunst. [Dissertation] (= Quellen zur Sprach- und Culturgeschichte der germanischen Völker 54). Straßburg: K.J. Trübner. https://archive.org/stream/bub_gb_g-gIAAAAQAAJ\#page/n23/ mode/2up, Zugriff: 15.4.2018.

Keel, William (2002). »Was ist eine Schnitzelbank? The Tradition behind the Popular German-American Sing-Along «. In: Missouri Folklore Society Journal 24, S. 21-35.

Keel, William (2003). »A German-American Icon: O, du schöne Schnitzelbank!« In: Yearbook of German-American Studies 38. Hg. v. dems. Lawrence: Society for German-American Studies, S. 221-253.

Keel, William (2009). »From Bänkelsang to Schnitzelbank: A Never-Ending Story. «In: Er ist ein wol gevriunder man: Essays in Honor of Ernst C. Dick on the Occasion of His Eightieth Birthday. Hg. v. Karen McConnell u. Winder McConnell. Hildesheim u.a.: Olms, S. 213-236.

Kohring, Jens (2000). »Brauchtum - der moralische Schatten«. In: Geheimsache Schnitzelbank. 1851-2001. Hg. v. Kultur-, Presse- und Touristikamt der Stadt Ellwangen. Ellwangen: o.V., S. 10-25.

Kohring, Jens / Nußbaumer, Wolfgang / Hurler, Joseph (2000). »Der Pennäler Schnitzelbank«. In: Geheimsache Schnitzelbank. 1851-2001. Hg. v. Kultur-, Presse- und Touristikamt der Stadt Ellwangen. Ellwangen: o.V., S. 165 u. 173.

Korson, George (1949). Pennsylvania Songs and Legends. Baltimore: Johns Hopkins University Press.

Mannhardt, Wilhelm (1855). »Der Kukuk.«In: Zeitschrift für Deutsche Mythologie und Sittenkunde 3, S. 209-298.

New York Times Immigration Explorer (2009). »Remade in America. The Newest Immigrants and their Impact «, https://archive.nytimes.com/www.nytimes.com/ interactive/2009/03/10/us/20090310-immigration-explorer.html?_r=0, Zugriff: 1.7.2018.

Nußbaumer, Wolfgang (2000). »Mission, Moritaten und Maskierte - Ein Blick in die Geschichte eines Geheimbundes«. In: Geheimsache Schnitzelbank. 1851-2001. Hg. v. Kultur-, Presse- und Touristikamt der Stadt Ellwangen. Ellwangen: o.V. S. 32-37.

Petzoldt, Leander (1978). Die freudlose Muse: Texte, Lieder und Bilder zum historischen Bänkelsang. Stuttgart: J.B. Metzler u. Carl Ernst Poeschel.

Röhrich, Lutz / Brednich, Rolf Wilhelm (1967). Deutsche Volkslieder. Texte und Melodien Bd. 2: Lieder aus dem Volksleben. Düsseldorf: Pädagogischer Verlag Schwann.

Seemann, Erich (1959). Korrespondenz zwischen Prof. Dr. Erich Seemann (DVA Freiburg) und Dr. Friedrich Carl Sarre (Rechtsanwalt) vom Januar 1959 zum Thema »Herkunft der Schnitzelbank«. Aus dem Archiv des ZPKM Freiburg, Gruppe XII: »Das ist kurz und das ist lang «. Kopie im Privatarchiv des Autors. 


\title{
Diskografie
}

Ames Brothers feat. Russ Morgan And His Orchestra (1949). »Oh, You Sweet One (The Schnitzelbank Song)«. Coral, 60065-B.

Blanc, Mel (1953). »Yah, das ist ein Christmas Tree«. Capitol, F2619-A.

Falco (1985). »Rock Me Amadeus (Extended Version)«. US-Version, A\&M, SP-12150.

Haley, Bill (1956). »Rockin' Rollin' Schnitzelbank«. Auf: Rockin' Around The World. Decca, DL 8692.

Heidelberg Quartette (1922). »Schnitzelbank«. Gennet, 4884-A.

Joe Biviano (1949). »Schnitzel Bank«. RCA Victor, 25-1129.

John Schmid (2004). »Schnitzelbank«. iTunes: https://itunes.apple.com/us/ album/in-dutch!/id571654998.

Lawrence Duchow (1951). »Oh, You Sweet One (The New Schnitzelbank Song)«. RCA Victor 25-1193.

Leighton Noble (1949). »Oh, You Sweet One (The Schnitzelbank Song)«. Vocalion, 55015-B.

Louis Bauer Quartett (1913). »Schnitzelbank«. Columbia, E1315.

Manhattan Quartet (1911). »Die Schnitzelbank«. Victor, C-10326.

Nebe Quartet (1913). »Ei du schöne Schnitzelbank«. Edison Blue Amberol, 26082.

Nena (1983). »99 Luftballons«. CBS, CBSA, 12.3060.

Rudy Vallee (1962). »Schnitzelbank«. Auf: Stein Songs. Decca, 74242.

Rammstein (1995a). »Rammstein«. Auf: Herzeleid. Motor, 529160-2.

Rammstein (1995b). »Heirate mich«. Auf: Herzeleid. Motor, 529160-2.

Six Fat Dutchmen (1956). "Schnitzelbank Polka«. RCA Victor, 47-6593-A.

The Andrew Sisters (1949). »Oh, You Sweet One (The Schnitzelbank Song)«. Decca, 24664-B.

The Weidler Brothers (vermutlich 1949). »The Schnitzelbank Polka«. Capitol, 78-108. Thurl Ravenscroft (1956). »Oh You Sweet One«. Bally, 1008-A.

Trio (1982). »Da Da Da Ich Lieb Dich Nicht Du Liebst Mich Nicht Aha Aha Aha«. Mercury, 6400544.

\begin{abstract}
The "Schnitzelbank « is a chain-song-type whose structure and rhyme scheme have been used in quite a number of US pop songs in the 20th century. Though the phenomenon of the Schnitzelbank is only little known throughout Germany, it seems to be ubiquitous in American popular culture, appearing in movies, cartoons and advertisements as well as in newspapers. The Schnitzelbank is based on three distinct European traditions: street ballads from the Middle Ages, ancient wedding customs of German fishermen in the Gdansk area as well as specific Shrovetide customs in the cities of Ellwangen (Germany) and Basel (Switzerland). The present study focuses on the varieties of the Schnitzelbank manifestations in the USA and offers data from archival research.
\end{abstract}

